Unfallchirurg 2010 $\cdot 113: 870$

DOI 10.1007/s00113-010-1869-6

Online publiziert: 10 . September 2010

๑) Springer-Verlag 2010

\author{
H. Siebert \\ Generalsekretär, Deutsche Gesellschaft für Orthopädie und Unfallchirurgie e.V., \\ Deutsche Gesellschaft für Unfallchirurgie e.V., Berlin
}

\title{
„Das Desaster von Dresden“
}

\section{Deutscher Ärztetag 2010}

Kommentar zum Beitrag

Tempka A (2010) Zankapfel Weiterbildungsordnung. Unfallchirurg 113:871-873

Selten haben wir Chirurgen so gespannt auf die Entscheidungen des Deutschen Ärztetages bzgl. Musterweiterbildungsordnung gewartet wie in diesem Jahr. Von chirurgischer Seite mit Einbindung aller Berufsverbände haben wir wichtige Anträge zur Änderung der Musterweiterbildungsordnung im Gebiet Chirurgie in wahrlich mühsamen Sitzungen erarbeitet und rechtzeitig der Weiterbildungskommission der Bundesärztekammer vorgelegt. Parallel liefen Abstimmungsgespräche mit Präsidenten der Ärztekammern und Ärztekammerdelegierten des Ärztetages, um die Musterweiterbildungsordnung den derzeitigen und künftigen Rahmenbedingungen in unserem Gebiet und den chirurgischen Fächern anzupassen. Dazu zählt für das Fach Orthopädie und Unfallchirurgie der Antrag, das fachgebundene Röntgen als Bestandteil der fachärztlichen Weiterbildung aufzunehmen, um zu garantieren, dass alle Fachärzte bei Niederlassung die Chance haben, Röntgenaufnahmen selbständig durchführen zu können. Voraussetzung ist natürlich die per Gesetz vorgeschriebene Schulung.

Daneben wurde die Zusammenlegung der Facharztsäulen Allgemeine Chirurgie und Viszeralchirurgie in eine gemeinsame Säule und darauf aufbauend eine Zusatzweiterbildungsqualifikation „Spezielle Viszeralchirurgie" beantragt.

Auch ein Antrag zum fachgebundenen MRT und weitere Anträge für das Gebiet Chirurgie wurden rechtzeitig den Gremien vorgelegt.
Was kam dabei heraus?

\section{- Nahezu alle Anträge wurden dem Vorstand der Bundesärztekammer zur weiteren Bearbeitung zugewiesen.}

Die für uns wichtigen Anträge wurden somit nicht entschieden. Der „Weg durch die Instanzen " muss nun erneut gegangen werden. Vielleicht entscheidet aber auch der Vorstand der Bundesärztekammer anders, wir wissen es nicht.

Weiterzubildende in Neu-Ulm werden teilweise andere Inhalte und Kenntnisse zur Erlangung ihres Facharztes Orthopädie und Unfallchirurgie haben als ihre Kolleginnen und Kollegen in Ulm. Links- und rechtsrheinisch bestehen unterschiedliche Bedingungen und Voraussetzungen zur Befugniserteilung im Fach Orthopädie und Unfallchirurgie. Die Weiterbildungsbefugniserteilung zum fachgebundenen Röntgen wird in vielen Kammern völlig unterschiedlich geregelt. In Berlin ist derzeit der Prozess, das Röntgen wieder zurück ins Fach zu holen, angeschoben worden. Viele von uns, die seit Jahren diese Kleinstaaterei im Bereich der Weiterbildung erleben, können nicht verstehen, dass im Zeitalter papierloser Informationstechnologie, europäischer Facharztexamina und Regulierung der Migration von Ärzten in Europa hier in Deutschland derart unverständliche, kleinstaatliche Regelungen existieren. Dies ist dem Föderalismus geschuldet, wie Almut Tempka in ihrem Artikel ausführt. Vielleicht wird nun der Einen oder dem Anderen etwas klarer, warum wir uns in Deutschland, wo dieses Recht an die einzelnen Ärztekammern delegiert wurde, schwerer mit einer einheitlichen Regelung tun als etwa die Kollegen in England, wo die Fachgesellschaften ,den Hut" für die Regelung der Weiterbildungsordnung aufhaben.

Dennoch: Die Juristen haben uns das bereits seit Jahrzehnten vorgemacht, in dem sie eine Kammer beauftragten, eine einheitliche Weiterbildungsordnung für alle Kammern zu schaffen. Warum kann dieses Modell nicht auch für uns Ärzte gelten? Bei allen guten Gründen, die von Frau Tempka stringent aufgezeigt wurden und von unseren Kollegen bei den Kammern mit Überzeugung vertreten werden, bei all diesen guten Gründen sollten wir dennoch dafür Sorge tragen, dass der Föderalismus nicht zum Selbstzweck wird. In der Gemeinsamen Weiterbildungskommission der chirurgischen Fachgesellschaften und Berufsverbände werden wir dieses Thema im Herbst aufgreifen und versuchen, Bewegung im Sinne einer bundeseinheitlichen Harmonisierung zu bringen. Zur Not wird uns eventuell hier die Politik unterstützen müssen.

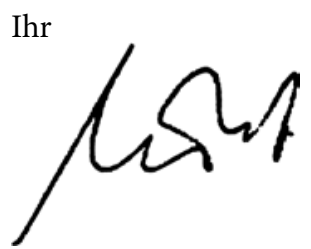

H. Siebert

\section{Korrespondenzadresse \\ Prof. Dr. H. Siebert}

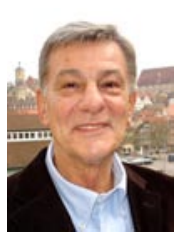

Generalsekretär Deutsche Gesellschaft für Orthopädie und Unfallchirurgie e.V., Deutsche Gesellschaft für Unfallchirurgie e.V. Luisenstr. 58, 10778 Berlin hsiebert@diaksha.de 